\title{
Efficient Lactic Acid Production from Dilute Acid Pretreated Lignocellulosic Biomass by a Synthetic Consortia of Engineered Pseudomonas Putida and Bacillus Coagulans
}

\section{Lihua Zou}

Nanjing Forestry University

Shuiping Ouyang

Nanjing Forestry University

Yueli Hu

Nanjing Forestry University

Zhaojuan Zheng

Nanjing Forestry University

Jia Ouyang ( $\square$ hgouyj@njfu.edu.cn )

Nanjing Forestry University https://orcid.org/0000-0002-3467-253X

\section{Research Article}

Keywords: Lignocellulosic hydrolysate, Detoxification, Pseudomonas putida, Bacillus coagulans, Microbial consortium, Lactic acid

Posted Date: September 29th, 2021

DOl: https://doi.org/10.21203/rs.3.rs-923947/v1

License: (a) This work is licensed under a Creative Commons Attribution 4.0 International License. Read Full License 


\section{Abstract \\ Background}

Lignocellulosic biomass is an attractive and sustainable alternative to petroleum-based feedstock for the production a range of biochemicals, and a pretreatment is generally regarded to be indispensable for its biorefinery. Nevertheless, various inhibitors that severely hindered the growth and fermentation of microorganisms were produced inevitably during the pretreatment of lignocellulose. Presently, a single microorganism that can tolerate toxic mixtures of pretreatment hydrolysate while effectively transforming sugar components into valuable compound is less well reported. Alternatively, microbial co-culture provides a simpler and more efficacious way to realize this goal via distributing metabolic tasks among proper strains.

\section{Results}

In this study, a novel synthetic microbial consortia, which is composed of a responsible for detoxification bacterium engineered Pseudomonas putida KT2440 and a lactic acid production specialist Bacillus coagulans NL01, was developed to directly produce lactic acid from high-toxic lignocellulosic hydrolysate. The engineered $P$. putida with deletion of sugar metabolism pathway was suggested to be unable to consume the major fermentable sugars of lignocellulosic hydrolysate, but can rapidly remove inhibitors in hydrolysate. With detoxification using engineered $P$. putida for $24 \mathrm{~h}$, the pretreated hydrolysate was fermented into $35.8 \mathrm{~g} / \mathrm{L}$ of lactic acid by $B$. coagulans with a yield of $90 \%$. The fermentation performance of microbial co-culture was significantly improved than that single culture of $B$. coagulans without lactic acid production.

\section{Conclusions}

The microbial coculture system constructed by this study demonstrated strong potential of the process for biosynthesis of valuable product from lignocellulosic hydrolysate containing high concentration of complex inhibitors by specifically recruited consortia of robust microorganisms with desirable characteristics and also provided a feasible and attractive method for bioconversion of lignocellulosic biomass to other valueadded biochemicals.

\section{Background}

Biochemicals produced from renewable resources through microbial fermentation is gaining rapidly growing interest due to the exhaustion of fossil fuels and the associated environmental issues [1]. Among these biochemicals, lactic acid and its derivatives are increasingly important building blocks, which have broad applications in food and chemical industries [2]. Currently, the biological approach for lactic acid production is very mature. But the cost of traditional substrates limits its use in the large-scale development of poly-lactic acid (PLA) as green alternative to petroleum-based plastics [3]. Lignocellulosic biomass 
mainly consisting of cellulose, hemicellulose and lignin, is the most abundant renewable carbon source on earth with low cost [4]. It is of great significance to realize the effective biotransformation from lignocellulose-derived sugars to lactic acid. However, owing to its recalcitrance and irregular bond structure, few microorganisms can directly utilize lignocellulose to a product of interest [5] and thus pretreatment steps are indispensable to improve its digestibility and release more fermentable sugars for microorganism fermentation [6]. Nevertheless, many pretreatment studies showed that various inhibitors that severely impede microbial growth and fermentation would be inevitably formed during the deconstruction of lignocellulose, mainly including organic acids, furan aldehydes and phenolic compounds [7]. These inhibitors constitute a severe obstacle to the efficient use of lignocellulosic sugars and the effective product of biochemicals. Therefore, developing low-cost approaches and microorganisms to overcome barriers of inhibitors from biomass for fermentation would contribute to the scale-up and commercialization of lignocellulosic biorefineries.

Currently, a single naturally microbial that can detoxify or tolerate the non-sugar ingredients of pretreatment hydrolysate while validly transforming the sugar mixtures into valuable products is less well reported [8]. Adaptive evolution was considered as a powerful tool to improve substrate utilization and tolerance to toxic of biochemicals producing bacteria [9]. A series of investigations have been conducted to achieve the production of biochemicals from the pretreatment hydrolysates by using evolved strains [10, 11]. However, the evolved process is time-consuming and labor-intensive, and the phenotypes of evolved strains are unstable. In the recent decades, genetically engineered hosts that can both tolerance inhibitors and produce a range of different biofuels or biochemicals from lignocellulose have been reported [12]. The common strategy is the introduction of inhibitor tolerant elements into product-generating microbes $[13,14]$. But owing to the complexity of lignocellulosic hydrolysates and the inherently challenging of engineering and optimizing heterogeneous functional modules in a single strain, low titers product and productivity were obtained by using mono-culture based on genetically engineered microorganisms [15]. Alternatively, microbial co-culture provides a more feasible and efficacious way to realize this objective [16].

In recent years, synthetic microbial consortium has garnered considerable interest especially in the field of industrial biotechnology because of its ability to perform complex biotransformation by division of labor [17]. Furthermore, microbial consortium synergies existed that can result in more efficient substrate utilization and increased product yield through functional modularity. Designed and established of microbial consortia with the capability of detoxification and biochemical production from pretreated lignocellulosic feedstock hold many appealing properties in practical lignocellulose biorefining. Zhu et al. developed an individual species co-culture system of Saccharomyces cerevisiae for bioethanol production from pretreated biomass. After process optimization, ethanol yield of co-culture was greatly improved than pure culture [18]. Biodetoxification of Amorphotheca resinae ZN1 provides a promising approach to remove inhibitors in hydrolysates. Dry dilute acid pretreatment corn stover are treated with A. resinae ZN1, subsequent cascade hydrolysis and fermentation, the production of gluconic acid and xylonic acid can be achieved by Gluconobacter oxydans [19]. A co-culture system of Acinetobacter baylyi ADP1 and Clostridium tyrobutyricum for removal of model inhibitors from mock hydrolysate and the production of hydrogen was successfully constructed [20]. These studies have illustrated the potential of co-culture strategy in the 
transformation of lignocellulosic biomass. Despite this progress, the microbial consortia in conversion of lignocellulosic hydrolysate still confronted with many difficulties, such as long detoxification time, considerable loss of sugars and inferior toxicity tolerance. The development and application of mixed cultures for pretreated biomass with high content of inhibitors has thus far been unachieved [21].

In this study, a novel synthetic microbial consortium composed of engineered P. putida KT2440 and $B$. coagulans NL01 directly converted undetoxified lignocellulosic hydrolysate to lactic acid was developed (Fig. 1). The sugar catabolism pathway of $P$. putida KT2440 was disabled by deleting the gene encoding glucose dehydrogenase, $g c d$, and gene encodes for glucose transporter, gtsABCD operon from its genome. The engineered strain could simultaneously digest multiple fermentation inhibitors in lignocellulosic hydrolysate while preserving the sugars for subsequent fermentation. B. coagulans utilize sugars reserved in the hydrolysate to produce lactic acid. The successful construction of the co-cultivation system suggests a promising route towards the development and utilization of lignocellulosic feedstock.

\section{Results And Discussion}

Undetoxified corn stover hydrolysate fermentation by B. coagulans

B. coagulans as a potential industrial lactic acid producing strain has been received widespread attention. It could effectively convert various monosaccharides (pentose and hexose) derived from lignocellulose into high optical purity L-lactic acid through homolactic fermentation under thermophilic conditions [22]. To exploit the effective utilization of lignocellulosic feedstocks by $B$. coagulans, the undetoxified dilute acid pretreatment corn stover hydrolysate consisting of glucose $(22.2 \mathrm{~g} / \mathrm{L})$, xylose $(122 \mathrm{~g} / \mathrm{L})$, arabinose $(\sim 16$ $\mathrm{g} / \mathrm{L})$, five carbohydrate degradation products, ten phenolic compounds and other unidentified inhibitors (Table 1 ) was used in this work. The hydrolysate was diluted to different concentrations $(20 \%, 30 \%$ and $40 \%$ $(\mathrm{v} / \mathrm{v}))$ and then directly employed for lactic acid fermentation by B. coagulans NL01. 
Table 1

Composition of sugars, weak acids, furans, and phenolic compounds present in concentrated undetoxified corn stover dilute-acid pretreatment hydrolysate.

\begin{tabular}{|lll|}
\hline & products & concentration \\
\hline Sugars $(\mathrm{g} / \mathrm{L})$ & Glucose & $22.36 \pm 0.24$ \\
\hline & Xylose & $121 \pm 1.08$ \\
\hline Weak acids $(\mathrm{g} / \mathrm{L})$ & Arabinose & $15.20 \pm 0.28$ \\
\hline & Formic acid & $1.35 \pm 0.24$ \\
\hline Furans $(\mathrm{g} / \mathrm{L})$ & Acetic acid & $14.50 \pm 0.25$ \\
\hline & Levulinic acid & $2.96 \pm 0.05$ \\
\hline Fhenolic compounds $(\mathrm{mg} / \mathrm{L})$ & Furfural & $0.4 \pm 16$ \\
\hline & H'4-Dihydroxybenzoic acid & $34.25 \pm 6.02$ \\
\hline 4-hydroxybenzoic acid & $41.34 \pm 0.99$ \\
\hline Vanillic acid & $66.35 \pm 5.33$ \\
\hline Syringic acid & $24.36 \pm 1.60$ \\
\hline 4-hydroxybenzaldehyde & $59.35 \pm 1.01$ \\
\hline Vanillin & $86.65 \pm 2.03$ \\
\hline P-coumaric acid & $133.90 \pm 2.64$ \\
\hline Syringaldehyde & $55.29 \pm 2.21$ \\
\hline Ferulic acid & $214.20 \pm 0.99$ \\
\hline Cinnamic acid & 0 \\
\hline Total phenolics & 5.33 \\
\hline & & \\
\hline & & \\
\hline
\end{tabular}

The growth and lactic acid fermentation capacity of $B$. coagulans in fermentation medium containing different concentrations of pretreatment hydrolysate were evaluated. As illustrated in Fig. $2 a$ and $2 b$, the growth and xylose utilization of $B$. coagulans decreased as the concentration of the hydrolysate was elevated. At a concentration of $20 \%(\mathrm{v} / \mathrm{v})$ hydrolysate with $31.93 \mathrm{~g} / \mathrm{L}$ monomeric sugars, the bacteria showed obvious growth after an initial $12 \mathrm{~h}$ lag phase. Which can completely uptake the primary monosaccharides composed of xylose, glucose and arabinose within $72 \mathrm{~h}$, and achieve the maximum $\mathrm{OD}_{600}$ 6.6. Whereas, the growth and lactic acid fermentation of the $B$. coagulans were completely ceased at a higher hydrolysate concentration $(30 \%(\mathrm{v} / \mathrm{v}))$. Even after prolonged fermentation time to $96 \mathrm{~h}$, the growth of $B$. coagulans was still seriously hindered. The same results were observed with $40 \%(\mathrm{v} / \mathrm{v})$ hydrolysate. 
These results demonstrated that $B$. coagulans had a certain tolerance to hydrolysate, but the tolerance ability of strain was not high. It was merely suited for hydrolysate with low inhibitors and low corresponding sugar concentration. It is well known that substrate titer is one of vital factors impacting the final product concentration [23]. However, the concentration of inhibitors in the hydrolysate would enhance with the increase of the concentration of sugar. Furthermore, synergistic effects of between inhibitors under high contents render of lignocellulosic hydrolysates more toxic. The poor fermentation performance indicated that the tolerance of $B$. coagulans to higher concentration of hydrolysate was very inferior. These features limit the potential of using it as a platform strain to handle the carbohydrate products of lignocellulosic waste [24]. To promote the effective conversion of lignocellulosic sugars in hydrolysate, the introduction of an outstanding detoxified strain capable of transforming and degrading inhibitors while preserving the sugars is highly desirable for removing the inhibitory and toxic compounds to improve the fermentation of B. coagulans.

\section{Metabolic engineering of P. putida to block sugar metabolism and growth characterization of knockout strains}

P. putida KT2440 has emerged as a promising candidate for a future lignocellulose-based biotechnology process due to its outstanding toxicity tolerance to numerous by-products of biomass hydrolysis [25-27]. This was confirmed here by incubating of wild-type $P$. putida in LB medium supplemented with major inhibitors found in lignocellulosic hydrolysate (Fig. S1). Besides, it is a robust microorganism capable of natively catabolizing glucose other than toxins. Unfortunately, the excessive utilization of sugar during the detoxification will adversely affect subsequent fermentation and reduce process efficiency. With the intent of developing a prominent detoxified strain capable of removing inhibitors in hydrolysate and retaining monomeric sugars, we engineered $P$. putida to delete its sugar metabolism. There are three glucose catabolism pathways in P. putida KT2440 (Fig. 3). The first route is that glucose is transported to cytoplasm from periplasm by means of an ATP dependent ABC transporter (PP_1015-PP_1018), encoded by the gts $A B C D$ operon and is then phosphorylated by glucokinase to glucose-6-phosphate and subsequently converted by glucose-6-phosphate dehydrogenase to 6-phosphogluconate. The second is a periplasmic oxidation pathway mediated by PQQ-dependent glucose dehydrogenase gcd (PP_1444). Glucose is oxidized to gluconate by gcd in the periplasm, which is then transported to the cytoplasm and subsequently phosphorylated, later reduced to 6-phosphogluconate. Besides, previous studies revealed that xylose is also oxidized to xylonate by $g c d$ [28]. The third route is the 2-ketogluconate loop, which involves the oxidation of gluconate to 2-ketogluconate. The resulting 2-ketogluconate is imported into the cytoplasm via the outer membrane and subsequently phosphorylated, in which the oxidation pathway and the direct phosphorylation route converge at the node of 6-phosphogluconate and that function simultaneously [28, 29]. Accordingly, the $g c d$ and $g t s A B C D$ were selectively removed.

The growth and the consumption of fermentable sugars of $P$. putida and its knockout strains were characterized. As a reference, the cultivation of $P$. putida KT2440 in M9 minimal medium using glucose or xylose as a carbon source was performed. As shown in Fig. 4 a, about $5 \mathrm{~g} / \mathrm{L}$ of glucose can be used up by $P$. putida KT2440 in $6 \mathrm{~h}$, and reach a remarkable maximum cell $\mathrm{OD}_{600}$ of 4.5 . However, when the carbon source of $P$. putida KT2440 was switched to xylose, although the xylose concentration was significantly 
reduced and dropped to 0 at $30 \mathrm{~h}$, the growth of the strain was not detected (Fig. 4c). The consumed xylose was oxidized to xylonate instead of being used as a carbon source for strain growth. The results obtained are consistent with previous study [30]. The growth performance of the $P$. putida pK18MS- $\Delta g c d$ was evaluated under the same carbon source conditions. When incubating with glucose, P. putida KT2440 pK18MS- $\Delta g c d$ showed a significantly slower growth than wild type and the consumption rate of glucose was remarkably decreased, which was not completely consumed until $36 \mathrm{~h} \mathrm{(Fig.} 4 \mathrm{~b}$ ). The ability of the $P$. putida KT2440 pK18MS- $\Delta$ gcd to utilize xylose was also tested (Fig. 4d). No growth of strain and reduced sugar concentration can be examined during the entire cultivation. The deletion of gcd could greatly retard the glucose metabolism and completely hinder the consumption of xylose. These results suggested that gcd is of paramount importance for glucose and xylose uptake in P. putida.

The successful knockout strain $P$. putida KT2440 pK18MS- $\triangle g c d-\triangle g t s A B C D$ was verified using glucose or xylose as a substrate. As predicted, the strain did not illustrate any detectable growth and consumption of sugar during 4 days of the cultivation on glucose or xylose (Fig. 4e \& Fig. 4f). Removal of gcd and gtsABCD from $P$. putida KT2440's genome rendered it unable to utilize glucose and xylose. Biological detoxification of lignocellulosic hydrolysate has been proved to be a prospective approach for inhibitors removal and many microorganisms that either degrade or convert inhibitors have been identified. However, most of detoxified microorganisms consume main fermentable sugars, which causing a substantial loss of the sugar content in the lignocellulose hydrolysate. Efficient biological detoxification required microorganism that metabolized all inhibitors while preserving the sugar fraction intactly [31].

\section{Inhibitors tolerance analysis of engineered P. putida KT2440 pK18MS- $\Delta$ gcd- $\Delta$ gtsABCD}

The ability of engineered P. putida KT2440 pK18MS- $\triangle g c d-\triangle g t s A B C D$ to metabolize and convert common inhibitors encountered in lignocellulose hydrolysates was investigated. Firstly, the effect of acetate, which was formed as a major unwanted byproduct from the breakdown of hemicellulose, was tested by culturing cells in M9 minimal medium comprising different titers of sodium acetate ranging from $0-10 \mathrm{~g} / \mathrm{L}$. As presented in Fig. 5a, the knockout strain could still grow well with different concentrations of sodium acetate as a carbon source. The supplementation of high concentrations sodium acetate $(5 \mathrm{~g} / \mathrm{L}$ and 10 $\mathrm{g} / \mathrm{L}$ ) only slightly delayed the growth of cells in the early stage, but did not affect their complete catabolism by the strain. Besides, the maximum achieved biomass of the strain was positively correlated with the concentration of the initial substrate provided. With $1 \mathrm{~g} / \mathrm{L}$ sodium acetate, the maximum $\mathrm{OD}_{600} 0.77$ was detected. Using $5 \mathrm{~g} / \mathrm{L}$ sodium acetate, higher growth rate was measured. The cells reached a maximum $\mathrm{OD}_{600}$ of 3.1 at the highest applied $10 \mathrm{~g} / \mathrm{L}$ of sodium acetate. During cultivation, the consumption of sodium acetate at different concentrations was also indicated in Fig. $5 \mathrm{~b}$. Up to $10 \mathrm{~g} / \mathrm{L}$ sodium acetate could be totally depleted by engineered $P$. putida in $48 \mathrm{~h}$. Although acetate itself is not a fierce inhibitor, its elimination could lessen the whole toxicity of hydrolysate [20]. Similarly, the utilization of levulinic acid by the knockout strain was assessed. The knockout strain did not exhibit lag phase at the tested concentrations (Fig. 5c). The strain could grow rapidly with levulinic acid. A maximum $\mathrm{OD}_{600}$ of 2.5 could be achieved starting at $5 \mathrm{~g} / \mathrm{L}$ levulinic acid. Correspondingly, the consumption of levulinic acid can also be seen from Fig. $5 d$. Levulinic acid was rapidly consumed in a short period. Even though previous studies have reported that weak acids can inhibit cell growth and metabolism due to uncoupling and intracellular 
anion accumulation, we found that engineered $P$. putida grew well in the presence of carbohydrate derived acids [32]. FAL and HMF that commonly generated from high-temperature processing of lignocellulose are considered major inhibitors in microbial conversion processes [33]. The conversion performance of the engineered $P$. putida KT2440 pK18MS- $\triangle g c d-\triangle g t s A B C D$ towards furan aldehydes was examined. As shown in Fig. $5 \mathrm{e}$ and $5 \mathrm{f}$, the engineered strain could convert the representative concentrations of HMF and FAL into the corresponding carboxylic acids, 5-hydroxymethyl-2-furancarboxylic acid (HMFCA) and furoic acid (FA), which are less toxic. In addition, a different conversion profile by engineered $P$. putida was noticed. Compared with FAL, the conversion rate of HMF were faster within $12 \mathrm{~h}(0.847 \mathrm{mM} / \mathrm{h}$ for HMF and 0.557 $\mathrm{mM} / \mathrm{h}$ for $\mathrm{FAL}$ ). The complete conversion of HMF was observed at $12 \mathrm{~h}$ of cultivation, whereas full conversion of FAL was observed at $24 \mathrm{~h}$.

These results demonstrated that the genetically modified $P$. putida remained robust to the elimination and transformation of toxic inhibitors. It has extraordinary potential for the removal of inhibitors in the biomass pretreatment hydrolysate.

\section{Production of lactic acid from undetoxified corn stover hydrolysate by using synthetic consortia of engineered $P$. putida and B. coagulans}

Based on the above results, a co-cultivation reaction was performed for L-lactic acid production from undetoxified $30 \%(\mathrm{v} / \mathrm{v})$ real hydrolysate that has the same composition as described above. During coculture process, to gain detailed insights into the behavior of engineered $P$. putida KT2440 pK18MS- $\triangle$ gcd$\triangle g t s A B C D$ on the complex mixtures of the hydrolysate, the detoxification reaction of the hydrolysate was set up using $1 \mathrm{~g} / \mathrm{L}$ P. putida KT2440 pK18MS- $\triangle g c d-\triangle g t s A B C D$ and no detoxified strain was supplemented as control. The concentration changes of the substances (fermentable sugars, organic acids, furans and phenol compounds) before and after the reaction were detected. After $24 \mathrm{~h}$ reaction, the concentration of major sugars such as glucose, xylose and arabinose in lignocellulosic hydrolysate remained nearly constant under both conditions (Table S1). However, there are considerable difference in the degradation and conversion of inhibitors. In the presence of detoxified strain, $4.3 \mathrm{~g} / \mathrm{L}$ of acetic acid and $0.9 \mathrm{~g} / \mathrm{L}$ of levulinic acid could be completely metabolized in $12 \mathrm{~h}$. Besides, the removal rate of formic acid and HMF reached $73 \%$ and $100 \%$, respectively (Fig. 6a). Furthermore, the concentration changes of total phenol and several typical monophenol inhibitors were also quantified. As shown in Table S2 and Fig. 6c, we observed that the concentration of total phenol and monophenol inhibitors have enormous degrees of reduction. The detoxification for $24 \mathrm{~h}$ by $P$. putida KT2440 pK18MS- $\operatorname{loc}-\triangle g t S A B C D, 25 \%$ of total phenolic derivatives was found to be removed. This result is comparable to the electrochemical detoxification reported in the literature [23]. Additionally, most of the representative monophenol compounds especially ferulic acid, $p$ coumaric acid, vanillin, 4-hydroxybenzaldehyde and syringaldehyde could be rapidly degraded to very low concentration. A control experiment confirmed that the concentration of the organic acids, furan aldehydes (Fig. 6b), ten representative phenolic compounds (Fig. 6d) as well as total phenolic derivatives (Table S2) did not vary significantly in the absence of detoxified strain pK18MS- $\operatorname{loc}-\triangle g t s A B C D$ under the same investigated conditions. These results indicated that $P$. putida KT2440 pK18MS- $\triangle g c d-\triangle g t s A B C D$ is a superior biological detoxification strain, which can effectively eliminate the toxic compounds in 
depolymerized lignocellulose streams while retaining the hexose and pentose sugars in the hydrolysate for subsequent lactic acid fermentation.

The feasibility of detoxified hydrolysate for lactic acid fermentation by $B$. coagulans was investigated. As shown in Fig. 7, the hydrolysate treated by $P$. putida KT2440 pK18MS- $\triangle g c d-\triangle g t s A B C D$ containing glucose $(6.1 \mathrm{~g} / \mathrm{L})$, xylose (about $35 \mathrm{~g} / \mathrm{L}$ ) as well as arabinose $(4.5 \mathrm{~g} / \mathrm{L})$ could be effectively consumed by fermentation of strain $B$. coagulans within $96 \mathrm{~h}$, resulting in a lactic acid production of $35.8 \mathrm{~g} / \mathrm{L}$ and a corresponding yield of $90 \%$. However, the hydrolysate of the identical concentration that has not been detoxified by P. putida KT2440 pK18MS- $\triangle g c d-\triangle g t s A B C D$ has neither the consumption of sugar nor the production of lactic acid was observed (Fig. 2). As anticipated, the degradation and transformation of diverse inhibitors by $P$. putida in hydrolysate greatly decrease the inhibitors concentration and contribute to the broth less toxic for fermentation. The results suggested that the detoxification of $P$. putida was extremely beneficial for improving the utilization of sugar and lactic acid fermentation by $B$. coagulans in the hydrolysate with high content of complex inhibitors. Additionally, which also suggested that the coculture system was more advantageous than mono-culture fermentation for the production of biochemicals from highly toxic lignocellulosic hydrolysate.

\section{Comparison of lactic acid production from lignocellulosic biomass with other studies}

To date, extensive investigations have been conducted to realize the production of lactic acid from lignocellulosic biomass (Table 2). The production of lactic acid from the pretreated wheat straw hydrolysate by an adapted $B$. coagulans was reported. $35.5 \mathrm{~g}$ of lactic acid was obtained from $80 \mathrm{~g}$ wheat straw with a yield of $70.9 \%$ [34]. Besides, to reduce processing costs as well as simplification of the process, one genetically engineered Bacillus subtilis capable of both degrading the cellulose and producing lactic acid was developed. Nevertheless, the recombinant cellulolytic $B$. subtilis suffered from low titers of lactic acid [35]. Low efficiency was main barriers for lactic acid production from lignocellulosic biomass. Currently, most of studies on lactic acid production from lignocellulosic biomass commonly use the simultaneous saccharification and fermentation (SSF) method. Cui et al. reported a mixed cultures of Lactobacillus rhamnosus and Lactobacillus brevis for bioconversion corn stover into lactic acid through SSF process [36]. Although relatively high productivity of lactic acid was obtained, which require the addition of high-cost cellulase. Recently, consolidated bioprocesses (CBP) targeting lactic acid production was exploited by using a synthetic microbial consortia of Trichoderma reesei and Lactobacillus pentosus. Ultimately, $34.7 \mathrm{~g} / \mathrm{L}$ and $19.8 \mathrm{~g} / \mathrm{L}$ of lactic acid can be obtained from $5 \%(\mathrm{w} / \mathrm{w})$ microcrystalline cellulose and pretreated beech wood, respectively [37]. Despite intensive research efforts spanning years, as an immature but promising technology, there are limit reports on the conversion of lignocellulose by microbial co-cultivation especially in lignocellulosic hydrolysates made up of numerous inhibitors and high concentration. In this work, we developed synthetic microbial consortia of $P$. putida and $B$. coagulans could achieve effective production of lactic acid from lignocellulosic hydrolysate with high inhibitors contents. The titer and yield of lactic acid attained by the coculture system were comparable to those previously reported. Although the results obtained by this approach are encouraging, the process regulation and optimization of microbial cocultivation are still essential in the future work, which probably promote the concentration and productivity of lactic acid to satisfy the requirement of practical lignocellulose biorefining. 
Table 2

Comparison of lactic acid production from lignocellulosic biomass with other studies.

\begin{tabular}{|c|c|c|c|c|c|c|}
\hline Strain & Strategy & Substrate & $\begin{array}{l}C_{\mathrm{LA}} \\
\max \\
\left(\mathrm{g} \mathrm{L}^{-}\right. \\
\left.{ }^{1}\right)\end{array}$ & $\begin{array}{l}\text { Yield } \\
(\%) /(g / g)\end{array}$ & $\begin{array}{l}\text { Productivity } \\
\left(\mathrm{g} \mathrm{L}^{-1} \mathrm{~h}^{-1}\right)\end{array}$ & References \\
\hline $\begin{array}{l}\text { B. coagulans } \\
\text { CC17A }\end{array}$ & $\begin{array}{l}\text { adaptive } \\
\text { evolution, SSF }\end{array}$ & wheat straw & 35.5 & 70.9 & n.a. & 34 \\
\hline B. subtilis & $\begin{array}{l}\text { over- } \\
\text { expression of } \\
\text { endoglucanase } \\
\text { and knocked } \\
\text { out alpha- } \\
\text { acetolactate } \\
\text { synthase }\end{array}$ & cellulose & 4.1 & 63 & 0.03 & 35 \\
\hline $\begin{array}{l}\text { L. } \\
\text { rhamnosus/L. } \\
\text { brevis }\end{array}$ & co-culture, SSF & Corn stover & 20.95 & 0.70 & 0.58 & 36 \\
\hline $\begin{array}{l}\text { T. reesei/ L. } \\
\text { pentosus }\end{array}$ & CBP & $\begin{array}{l}\text { microcrystalline } \\
\text { cellulose }\end{array}$ & 34.7 & 62.4 & 0.16 & 37 \\
\hline $\begin{array}{l}\text { T. reesei/ L. } \\
\text { pentosus }\end{array}$ & CBP & Beech wood & 19.8 & 85.2 & 0.10 & 37 \\
\hline $\begin{array}{l}\text { P. putida/B. } \\
\text { coagulans }\end{array}$ & $\begin{array}{l}\text { Sequential co- } \\
\text { culture }\end{array}$ & $\begin{array}{l}\text { corn stover } \\
\text { hydrolysate }\end{array}$ & 35.8 & 0.90 & 0.37 & this study \\
\hline
\end{tabular}

\section{Conclusions}

In this study, a synthetic consortium was constructed for converting high-toxic lignocellulosic hydrolysate into lactic acid. The consortium consisted of engineered $P$. putida capable of consuming and transforming inhibitors while leaving the lignocellulosic sugars intact for subsequent fermentation and $B$. coagulans capable of utilizing fermentable sugars to produce lactic acid. Compared with fermentation of undetoxified hydrolysate by monoculture without lactic acid production, a microbial co-culture can generate $35.8 \mathrm{~g} / \mathrm{L}$ lactic acid with a yield of $90 \%$ from the equal titer of hydrolysate. Results herein affirmed the potential of using synthetic consortia to compartmentalize biological functions into proper strains for accomplishing complicated tasks that are difficult to achieve with monocultures.

\section{Methods}

\section{Materials}

Corn steep liquor was kindly donated by Shandong Xinpu Biotechnology Co., Ltd (Shandong, China). Concentrated undetoxified corn stover dilute-acid pretreatment hydrolysate was kindly provided by Shanghai Yigao Biotechnology Co., Ltd (Shanghai, China). P. putida KT2440 and B. coagulans NL01 were 
obtained from the Biochemical Engineering Research Institute of Nanjing Forestry University. Furfural (FAL) (99\%) and 5-Hydroxymethylfurfural (HMF) (99\%) were purchased from Sigma-Aldrich. Levulinic acid (> 97\%) was purchased from TCI (Japan).

\section{Plasmid and strain constructions}

The construction of $\mathrm{gcd}$ and $g t s A B C D$ mutants were conducted as described in our previous study [38]. Concisely, Genomic DNA of P. putida KT2440 was isolated using Bacteria Genomic DNA Extraction Kit (Takara, Japan). The upstream and downstream homologous arms of gcd and gtsABCD gene were separately PCR-amplified using genomic DNA from $P$. putida KT2440 as the template and the respective primers (Table S3). After gel purification, overlap extension PCR of gcd and gtsABCD homologous arms were executed using the primers $g c d$-up.f and $g c d$-down.r or gts $A B C D$-up.f and $g t s A B C D$-down.r. The resulting PCR products were recovered by Gel and PCR Clean-up system (Promega, USA) and ligated into pEASY-Blunt cloning vector to form new plasmids. The plasmids were digested with EcoR I and Bam $\mathrm{HI}$ and subsequently cloned into the corresponding restriction sites of the suicide vector pK18mobsacB to yield the recombinant plasmids pK18MS- $\operatorname{gcd}$ and pK18MS- $\triangle g t s A B C D$. Followed by electroporation plasmid pK18MS- $\Delta g c d$ into electrocompetent $P$. putida KT2440. Single crossover and double-crossover transformants of $P$. putida KT2440 pK18MS- $\Delta$ gcd were selected separately on LB plate with $50 \mu \mathrm{g} / \mathrm{mL}$ kanamycin and LB agar plate supplemented with $15 \%(\mathrm{w} / \mathrm{v})$ sucrose incubated at $30{ }^{\circ} \mathrm{C}$. The correct doublecrossover recombinant of $P$. putida KT2440 pK18MS- $\operatorname{gcd}$ as starting strain was used for further construction of $g t s A B C D$ knockout bacteria. The knockout process is the same as described above.

\section{The growth and transformation of sugars, organic acids and furan aldehydes by $P$. putida}

P. putida KT2440 and its knockout strains were routinely pre-cultured overnight in $5 \mathrm{~mL}$ of Luria Broth (LB) medium at $30{ }^{\circ} \mathrm{C}$ and $200 \mathrm{rpm}$. Then, the overnight cultures were collected by centrifuging at $6000 \mathrm{~g}$ for 5 min and supernatants were removed. The recovered cells were washed for twice with $0.85(\mathrm{w} / \mathrm{v}) \mathrm{NaCl}$. Followed by cells were inoculated at $\mathrm{OD}_{600}$ of $0.1-0.2$ in 250-ml shake-flask with $25 \mathrm{~mL}$ of M9 minimal medium [28] containing different substrates. The concentration of added glucose and xylose was depicted in the text or respective figure caption. In addition, acetate, levulinic acid, FAL, and HMF were added to the medium at specified concentrations, respectively. After addition of the inhibitors, the initial $\mathrm{pH}$ of the medium was adjusted to 7.0 using $\mathrm{NaOH}$ solution.

\section{Biodetoxification of lignocellulosic hydrolysate by engineered $P$. putida}

The engineered $P$. putida KT2440 pK18MS- $\operatorname{gcd}-\triangle g t s A B C D$ was pre-activated overnight. Then, $2 \%$ seed cultures were inoculated into $200 \mathrm{~mL}$ LB medium in $1 \mathrm{~L}$ shake flask incubated for around $12 \mathrm{~h}$, the cells were centrifuged (6000 $\mathrm{g}, 5 \mathrm{~min}$ ) and pellets were washed with $0.85(\mathrm{w} / \mathrm{v}) \mathrm{NaCl}$ solution twice. Subsequent cells harvested are used for biodetoxification. The biodetoxification was performed in an Erlenmeyer flask with $50 \mathrm{~mL}$ pretreated hydrolysate-containing fermentation medium ( $\mathrm{pH}$ 7.0) without nitrogen source supply, and $1 \mathrm{~g} / \mathrm{L}$ inoculum of $P$. putida KT2440 pK18MS- $\Delta g c d-\triangle g t s A B C D$ cells at $30^{\circ} \mathrm{C}, 200 \mathrm{rpm}$ under aerobic conditions. 


\section{Fermentation experiments of B. coagulans strain}

B. coagulans NL01 was used for lactic acid fermentation. Typically, the preculture of $B$. coagulans NL01 was conducted at $50{ }^{\circ} \mathrm{C}$ and $150 \mathrm{rpm}$ for $12 \mathrm{~h}$ in seed medium, which containing $(\mathrm{g} / \mathrm{L})$ : Xylose 20, Yeast extract 1 , Corn steep powder $2.5, \mathrm{NH}_{4} \mathrm{Cl} 1, \mathrm{MgSO}_{4} 0.2, \mathrm{CaCO}_{3} 10$, and then transferred into corresponding hydrolysate-containing fermentation medium with a $10 \%(\mathrm{v} / \mathrm{v})$ inoculum for lactic acid production. Fermentation experiments were performed in $150-\mathrm{mL}$ shake-flask containing $50 \mathrm{~mL}$ of fermentation medium. The samples were withdrawn at regular intervals for determining the amount of residual sugars and lactic acid produced during the reaction. The hydrolysate-containing medium used for $B$. coagulans fermentation was prepared as follows: concentrated undetoxified corn stover dilute-acid pretreatment hydrolysate was neutralized ( $\mathrm{pH}$ 6.5), then the hydrolysate was added to the fermentation medium (Ouyang et al. [34]) of B. coagulans deprived of carbon source.

\section{The process for production of lactic acid from lignocellulosic hydrolysate by bacteria-bacteria co-culture}

A two-stage biological process for the detoxification of hydrolysate and the generation of lactic acid was performed as follows. The detoxification using $P$. putida KT2440 pK18MS- $\triangle g c d-\triangle g t s A B C D$ was conducted at $30{ }^{\circ} \mathrm{C}$ with $200 \mathrm{rpm}$ for $24 \mathrm{~h}$ under aerobic conditions to degrade and convert inhibitors of hydrolysate, the $\mathrm{pH}$ was monitored and adjusted to 7.2 with $5 \mathrm{M} \mathrm{HCl}$. Followed by $B$. coagulans cells at $10 \%$ of the inoculum, organic nutrients (yeast extract $(2.5 \mathrm{~g} / \mathrm{L})$ and corn steep power $(1.2 \mathrm{~g} / \mathrm{L})$ ) as well as $\mathrm{CaCO}_{3}$ (about half of the total sugars concentration) were added to the hydrolysate for lactic acid fermentation. The reaction was shaken for further hours in the incubator at $50^{\circ} \mathrm{C}, 150 \mathrm{rpm}$.

\section{Analytic methods}

All the concentrations of the monomeric sugars, organic acids, furan aldehydes and lactic acid were detected by Agilent 1260 HPLC system with reflective index detector. The analysis was conducted using a column of Aminex HPX-87H (Bio-Rad, USA) operated at $55^{\circ} \mathrm{C}$ and the eluent flow rate was $0.6 \mathrm{~mL} / \mathrm{min}$ with $5 \mathrm{mM} \mathrm{H}_{2} \mathrm{SO}_{4}$, unless otherwise stated. The concentrations of phenolic inhibitors were determined as previously reported by Ouyang et al [34]. The cell growth was monitored turbidimetrically at optical density of $600 \mathrm{~nm}\left(\mathrm{OD}_{600}\right)$. When sugar and sugar acid are concomitantly presented in medium, the concentration of monosaccharide was detected according to the procedures described by Han et al. [39].

\section{Abbreviations}

FAL: Furfural; HMF: 5-Hydroxymethylfurfural; FA: Furoic acid; HMFCA: 5-Hydroxymethyl-2-furancarboxylic acid; gcd. glucose dehydrogenase; gtsABCD. glucose transporter; $\mathrm{NaOH}$ : Sodium hydroxide; SSF: Simultaneous saccharification and fermentation.

\section{Declarations}

\section{Ethics approval and consent to participate}


Not applicable.

\section{Consent for publication}

Not applicable.

\section{Availability of data and materials}

All data generated or analyzed during this study are included in this published article.

\section{Competing interests}

The authors declare that they have no competing interests.

\section{Funding}

This study was supported by the National Key Research and Development Program of China (2018YFA0902200), and the National Natural Science Foundation of China (22078163).

\section{Authors' contributions}

All authors contributed to the conception and planning of the study. LZ performed experiments, data analysis and prepared the manuscript. SOY and YH helped to perform the research. ZZ and JOY helped to guide the writing and revise the manuscript. All authors read and approved the final manuscript.

\section{Acknowledgements}

We thank Shandong Xinpu Biotechnology Co., Ltd (Shandong, China) for kindly providing corn steep liquor.

\section{References}

1. Himmel ME, Ding S, Johnson DK, Adney WS. Biomass Recalcitrance: Engineering Plants and Enzymes for Biofuels Production. Science. 2007;315:804-7.

2. Abdel-Rahman MA, Sonomoto K. Opportunities to overcome the current limitations and challenges for efficient microbial production of optically pure lactic acid. J Biotechnol. 2016;236:176-92.

3. Lu J, Lv Y, Qian X, Jiang Y, Wu M, Zhang W, et al. Current advances in organic acid production from organic wastes by using microbial co-cultivation systems. Biofuels Bioprod Biorefin. 2020;14:481-92.

4. Yang $H$, Yan $R$, Chen $H$, Lee DH, Zheng C. Characteristics of hemicellulose, cellulose and lignin pyrolysis. Fuel. 2007;86:1781-8.

5. Li H, Chen X, Ren J, Deng H, Peng F, Sun R. Functional relationship of furfural yields and the hemicellulose-derived sugars in the hydrolysates from corncob by microwave-assisted hydrothermal pretreatment. Biotechnol Biofuels. 2015;8:127.

6. Chandel AK, Garlapati VK, Singh AK, Antunes FAF, da Silva SS. The path forward for lignocellulose biorefineries: Bottlenecks, solutions, and perspective on commercialization. Bioresour Technol. 
2018;264:370-81.

7. Singh B, Verma A, Pooja, Mandal PK, Datta S. A biotechnological approach for degradation of inhibitory compounds present in lignocellulosic biomass hydrolysate liquor using Bordetella sp. BTIITR Chem Eng J. 2017;328:519-26.

8. Singh A, Bedore SR, Sharma NK, Lee SA, Eiteman MA, Neidle EL. Removal of aromatic inhibitors produced from lignocellulosic hydrolysates by Acinetobacter baylyi ADP1 with formation of ethanol by Kluyveromyces marxianus. Biotechnol Biofuels. 2019;12:91.

9. Sandberg TE, Salazar MJ, Weng LL, Palsson BO, Feist AM. The emergence of adaptive laboratory evolution as an efficient tool for biological discovery and industrial biotechnology. Metab Eng. 2019;56:1-16.

10. Shui ZX, Qin H, Wu B, Ruan Z, yong, Wang L, Tan FR, et al. Adaptive laboratory evolution of ethanologenic Zymomonas mobilis strain tolerant to furfural and acetic acid inhibitors. Appl Microbiol Biotechnol. 2015;99:5739-48.

11. Wang X, Khushk I, Xiao Y, Gao Q, Bao J. Tolerance improvement of Corynebacterium glutamicum on lignocellulose derived inhibitors by adaptive evolution. Appl Microbiol Biotechnol. 2018;102:377-88.

12. Wang S, Sun X, Yuan Q. Strategies for enhancing microbial tolerance to inhibitors for biofuel production: A review. Bioresour Technol. 2018;258:302-9.

13. Jiménez-Bonilla P, Zhang J, Wang Y, Blersch D, de-Bashan LE, Guo L, et al. Enhancing the tolerance of Clostridium saccharoperbutylacetonicum to lignocellulosic-biomass-derived inhibitors for efficient biobutanol production by overexpressing efflux pumps genes from Pseudomonas putida. Bioresour Technol. 2020;312:123532.

14. Wang X, Yomano LP, Lee JY, York SW, Zheng H, Mullinnix MT, et al. Engineering furfural tolerance in Escherichia coli improves the fermentation of lignocellulosic sugars into renewable chemicals. Proc Natl Acad Sci U S A. 2013;110:4021-6.

15. Roell GW, Zha J, Carr RR, Koffas MA, Fong SS, Tang YJ. Engineering microbial consortia by division of labor. Microb Cell Fact. 2019;18:35.

16. Jiang Y, Wu R, Zhou J, He A, Xu J, Xin F, et al. Recent advances of biofuels and biochemicals production from sustainable resources using co-cultivation systems. Biotechnol Biofuels. 2019;12:155.

17. Qian X, Chen L, Sui Y, Chen C, Zhang W, Zhou J, et al. Biotechnological potential and applications of microbial consortia. Biotechnol Adv. 2020;40:107500.

18. Zhu JQ, Li X, Qin L, Li WC, Li HZ, Li BZ, et al. In situ detoxification of dry dilute acid pretreated corn stover by co-culture of xylose-utilizing and inhibitor-tolerant Saccharomyces cerevisiae increases ethanol production. Bioresour Technol. 2016;218:380-7.

19. Hou W, Zhang M, Bao J. Cascade hydrolysis and fermentation of corn stover for production of high titer gluconic and xylonic acids. Bioresour Technol. 2018;264:395-9.

20. Kannisto MS, Mangayil RK, Shrivastava-Bhattacharya A, Pletschke BI, Karp MT, Santala VP. Metabolic engineering of Acinetobacter baylyi ADP1 for removal of Clostridium butyricum growth inhibitors produced from lignocellulosic hydrolysates. Biotechnol Biofuels. 2015;8:198. 
21. Brethauer S, Studer MH. Consolidated bioprocessing of lignocellulose by a microbial consortium. Energy Environ Sci. 2014;7:1446-53.

22. Zheng Z, Jiang T, Zou L, Ouyang S, Zhou J, Lin X, et al. Simultaneous consumption of cellobiose and xylose by Bacillus coagulans to circumvent glucose repression and identification of its cellobioseassimilating operons. Biotechnol Biofuels. 2018;11:320.

23. Jiang Y, Xu B, Yan W, Liu J, Dong W, Zhou J, et al. Inhibitors tolerance analysis of Clostridium sp. strain LJ4 and its application for butanol production from corncob hydrolysate through electrochemical detoxification. Biochem Eng J. 2021;167:107891.

24. Jiang T, Qiao H, Zheng Z, Chu Q, Li X, Yong Q, et al. Lactic acid production from pretreated hydrolysates of corn stover by a newly developed Bacillus coagulans strain. PLoS ONE. 2016;11:1-13.

25. Jiménez Jl, Miñambres B, García JL, Díaz E. Genomic analysis of the aromatic catabolic pathways from Pseudomonas putida KT2440. Environ Microbiol. 2002;4:824-41.

26. Elmore JR, Dexter GN, Salvachúa D, O’Brien M, Klingeman DM, Gorday K, et al. Engineered Pseudomonas putida simultaneously catabolizes five major components of corn stover lignocellulose: Glucose, xylose, arabinose, p-coumaric acid, and acetic acid. Metab Eng. 2020;62:62-71.

27. Horlamus F, Wang Y, Steinbach D, Vahidinasab M, Wittgens A, Rosenau F, et al. Potential of biotechnological conversion of lignocellulose hydrolyzates by Pseudomonas putida KT2440 as a model organism for a bio-based economy. GCB Bioenergy. 2019;11:1421-34.

28. Dvořák P, de Lorenzo V. Refactoring the upper sugar metabolism of Pseudomonas putida for coutilization of cellobiose, xylose, and glucose. Metab Eng. 2018;48:94-108.

29. Del Castillo T, Ramos JL, Rodríguez-Herva JJ, Fuhrer T, Sauer U, Duque E. Convergent peripheral pathways catalyze initial glucose catabolism in Pseudomonas putida: Genomic and flux analysis. J Bacteriol. 2007;189:5142-52.

30. Wang Y, Horlamus F, Henkel M, Kovacic F, Schläfle S, Hausmann R, et al. Growth of engineered Pseudomonas putida KT2440 on glucose, xylose, and arabinose: Hemicellulose hydrolysates and their major sugars as sustainable carbon sources. GCB Bioenergy. 2019;11:249-59.

31. Wierckx N, Koopman F, Bandounas L, De Winde JH, Ruijssenaars HJ. Isolation and characterization of Cupriavidus basilensis HMF14 for biological removal of inhibitors from lignocellulosic hydrolysate. Microb Biotechnol. 2010;3:336-43.

32. Zhang L, Li X, Yong Q, Yang ST, Ouyang J, Yu S. Impacts of lignocellulose-derived inhibitors on I-lactic acid fermentation by Rhizopus oryzae. Bioresour Technol. 2016;203:173-80.

33. Guarnieri MT, Ann Franden M, Johnson CW, Beckham GT. Conversion and assimilation of furfural and 5-(hydroxymethyl)furfural by Pseudomonas putida KT2440. Metab Eng Commun. 2017;4:22-8.

34. Ouyang S, Zou L, Qiao H, Shi J, Zheng Z, Ouyang J. One-pot process for lactic acid production from wheat straw by an adapted Bacillus coagulans and identification of genes related to hydrolysatetolerance. Bioresour Technol. 2020;315:123855.

35. Zhang XZ, Sathitsuksanoh N, Zhu Z, Percival Zhang YH. One-step production of lactate from cellulose as the sole carbon source without any other organic nutrient by recombinant cellulolytic Bacillus 
subtilis. Metab Eng. 2011;13:364-72.

36. Cui F, Li Y, Wan C. Lactic acid production from corn stover using mixed cultures of Lactobacillus rhamnosus and Lactobacillus brevis. Bioresour Technol. 2011;102:1831-6.

37. Shahab RL, Luterbacher JS, Brethauer S, Studer MH. Consolidated bioprocessing of lignocellulosic biomass to lactic acid by a synthetic fungal-bacterial consortium. Biotechnol Bioeng. 2018;115:120715.

38. Zheng Z, Xu Q, Tan H, Zhou F, Ouyang J. Selective Biosynthesis of Furoic Acid From Furfural by Pseudomonas Putida and Identification of Molybdate Transporter Involvement in Furfural Oxidation. Front Chem. 2020;8:1-10.

39. Han J, Hua X, Zhou X, Xu B, Wang H, Huang G, et al. A cost-practical cell-recycling process for xylonic acid bioproduction from acidic lignocellulosic hydrolysate with whole-cell catalysis of Gluconobacter oxydans. Bioresour Technol. 2021;333:125157.

\section{Figures}

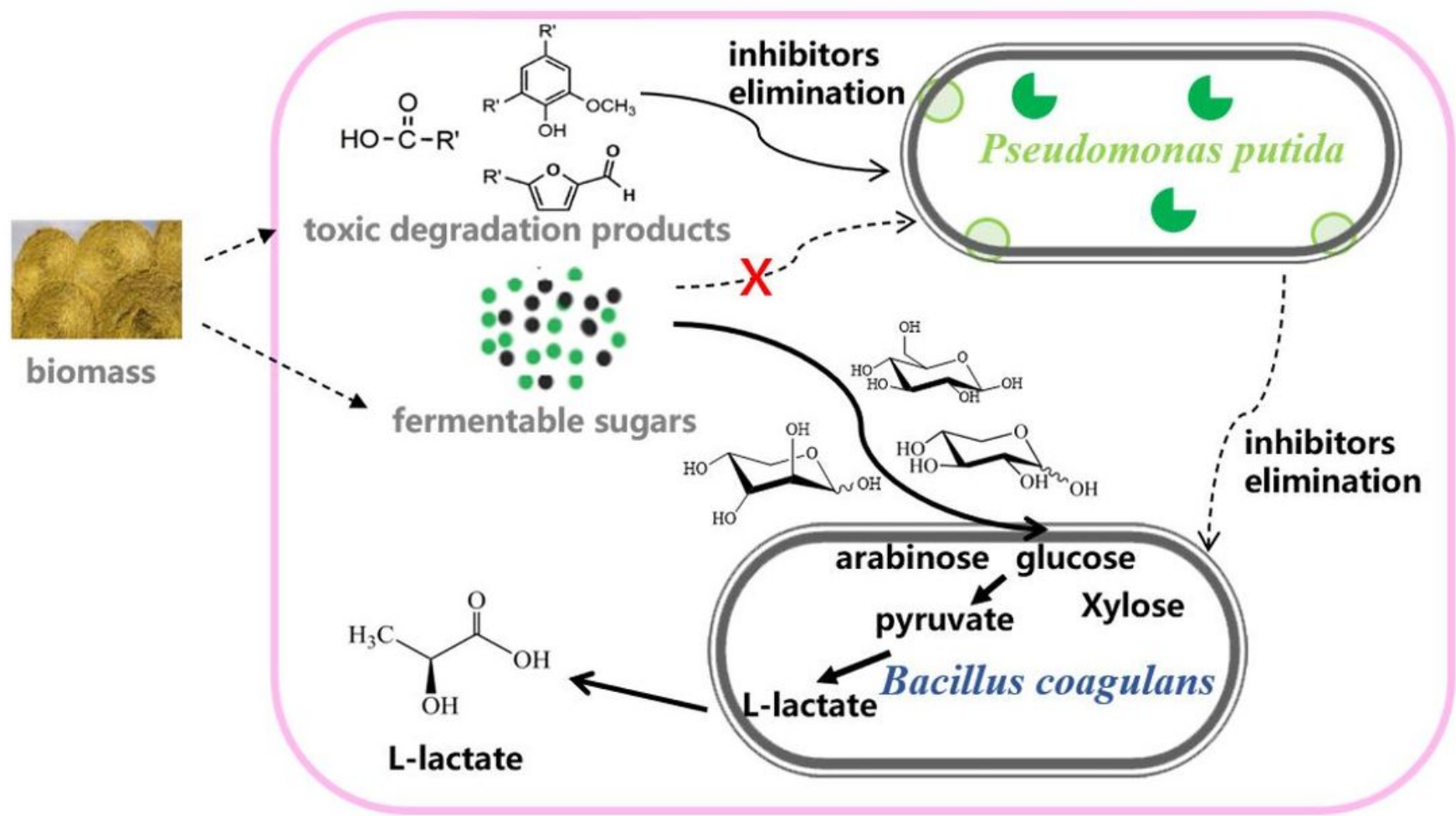

Figure 1

Construction diagram of the microbial coculture system for lactic acid production from lignocellulosic hydrolysate. (Toxic degradation products are mainly divided into three categories: furan aldehydes, organic acids and phenolic compounds; The green dots represent hexoses; The black dots indicate pentoses.) 
a

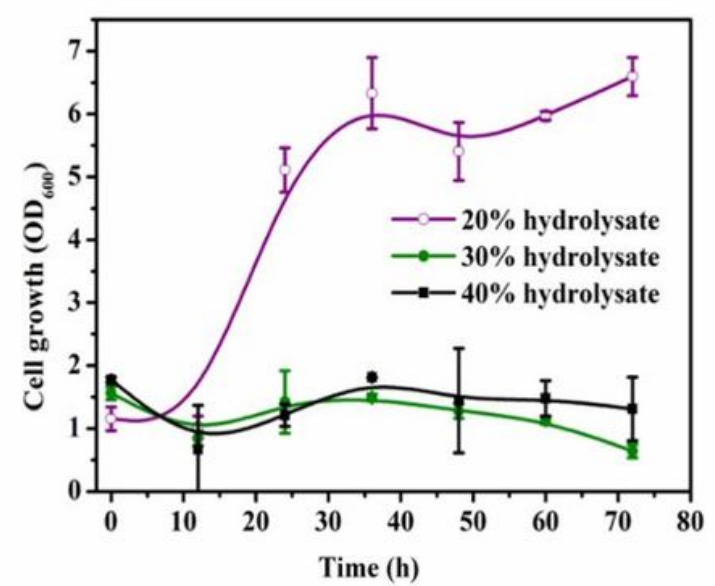

b

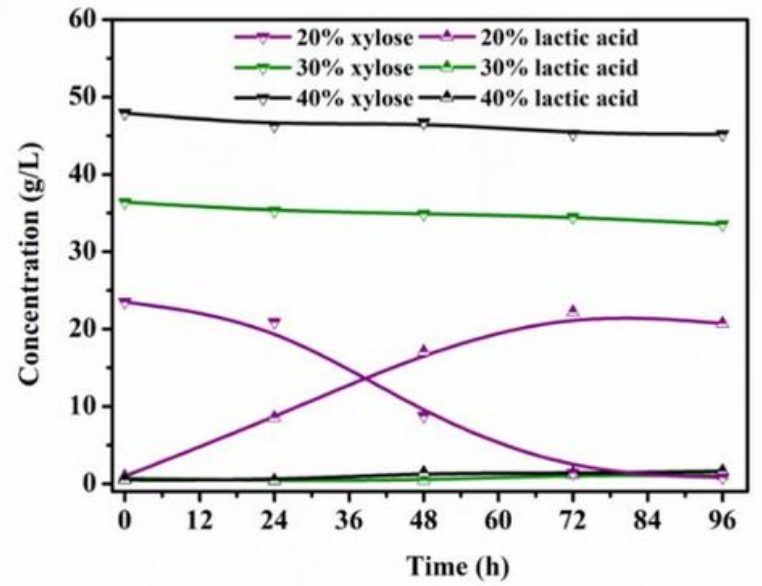

Figure 2

Undetoxified corn stover hydrolysate fermentation by B. coagulans NL01. Growth (a) and lactic acid fermentation (b) of B. coagulans NL01 with different initial concentrations of corn stover hydrolysate. Data shown as mean \pm SD from at least two independent experiments.

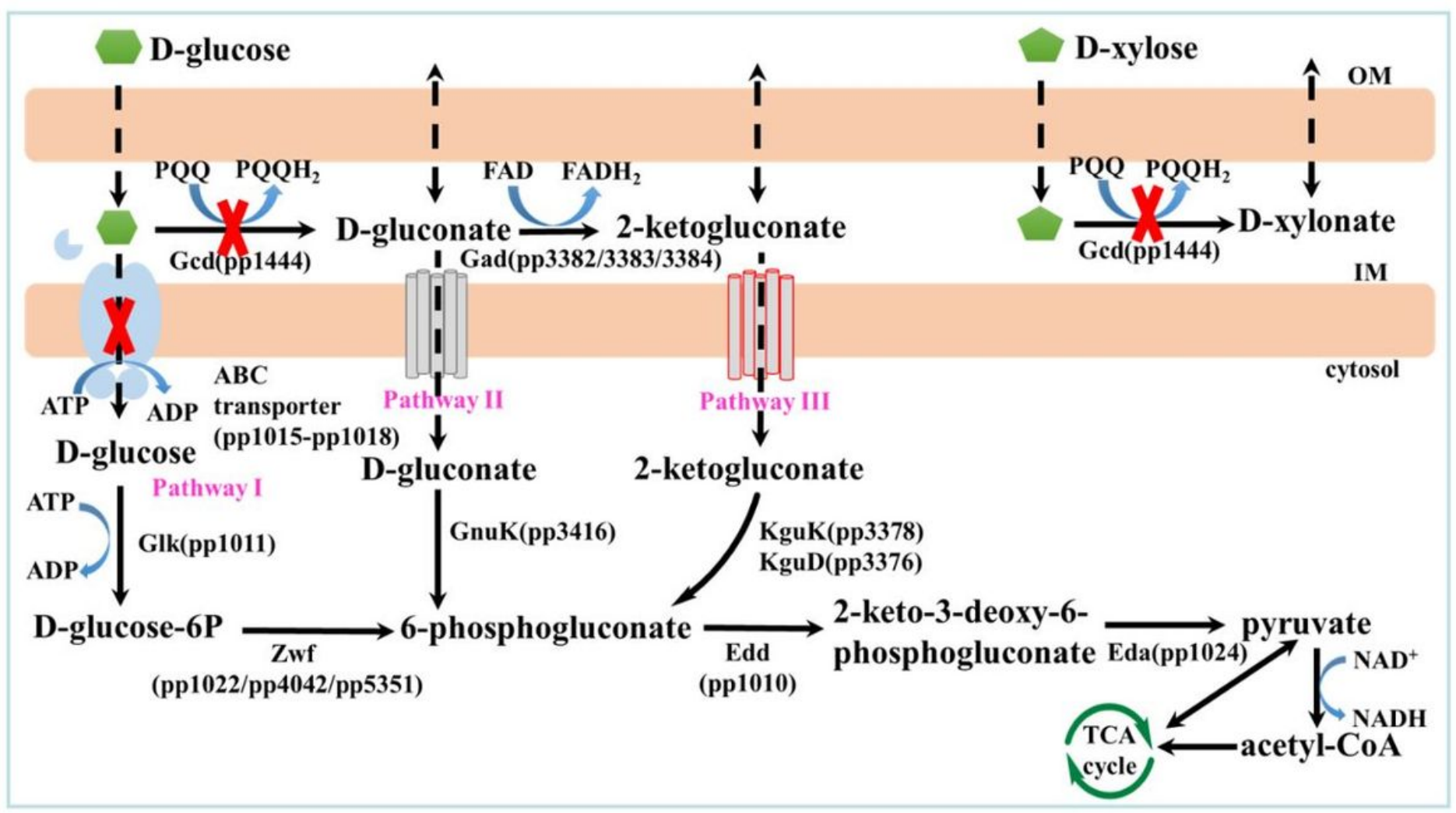

Figure 3

Schematic representation of glucose metabolism and xylose transformation in P. putida KT2440. Glucose catabolism in P. putida occurs through the simultaneous operation of three pathways that converge at the 
level of 6-phosphogluconate. D-xylose was oxidized to the dead-end product D-xylonate by the periplasmic glucose dehydrogenase Gcd instead of being used as a carbon source for growth. Abbreviations used for the metabolic intermediates and enzymes within the biochemical network are as follows: PQQ, pyrroloquinoline quinone; Gcd, glucose dehydrogenase; FAD, flavin adenine dinucleotide; Gad, gluconate dehydrogenase; Glk, glucokinase; GnuK, gluconate kinase; KguK, 2-ketogluconate kinase; KguD, 2ketogluconate-6-phosphatereductase; Zwf, glucose-6-phosphate dehydrogenase; Edd, 6-phosphogluconate dehydratase; Eda, 2-keto-3-deoxy-6-phosphogluconate aldolase; TCA cycle, tricarboxylic acid cycle; OM, (outer membrane); IM (inner membrane).

a

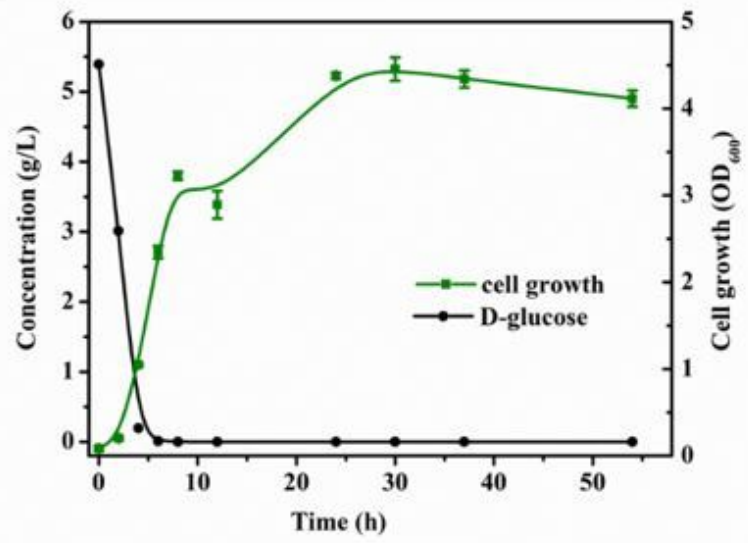

c

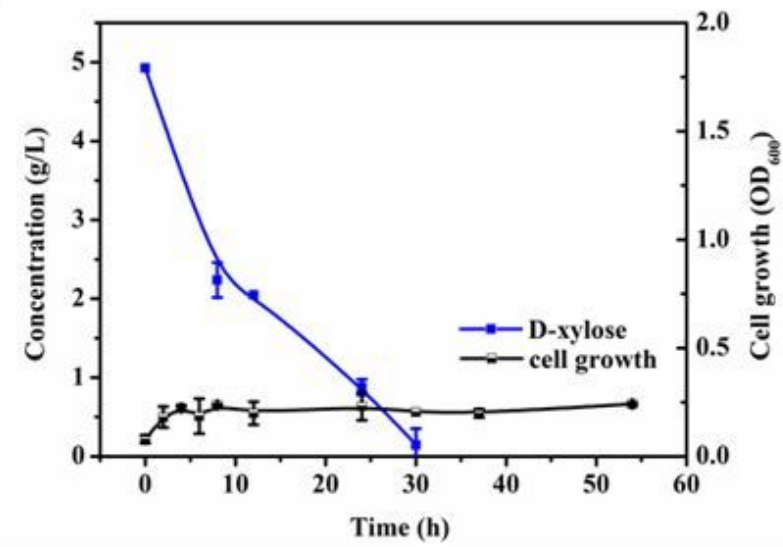

e

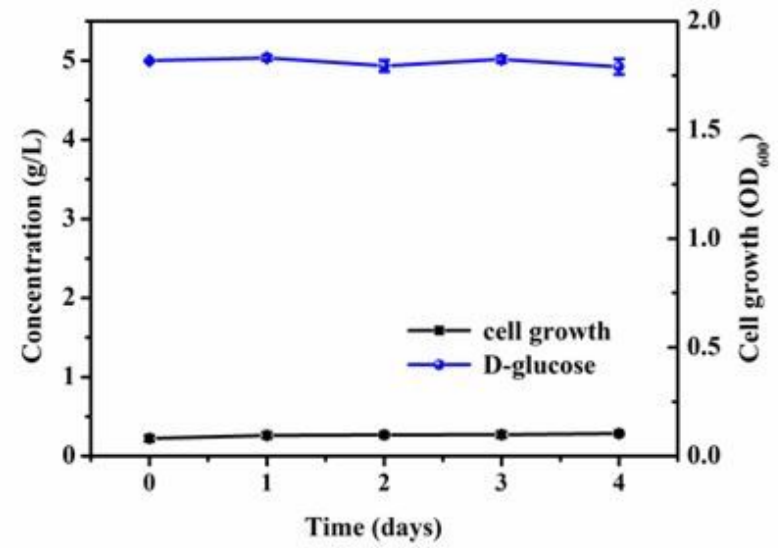

b

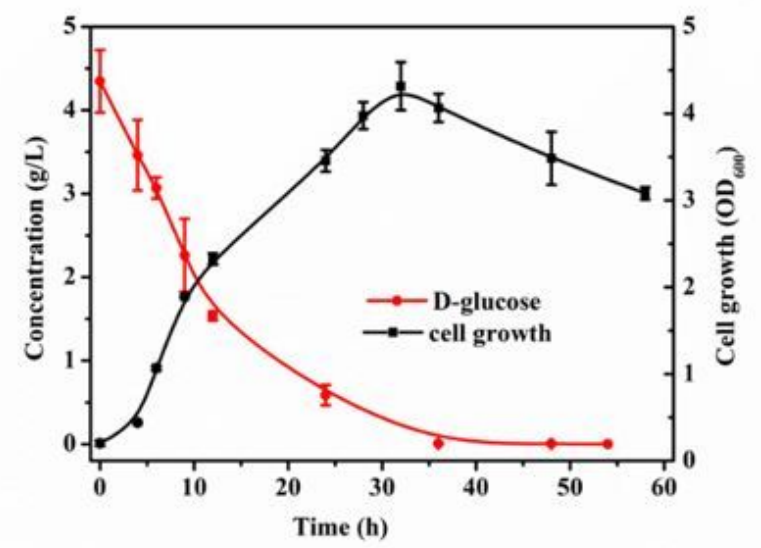

d

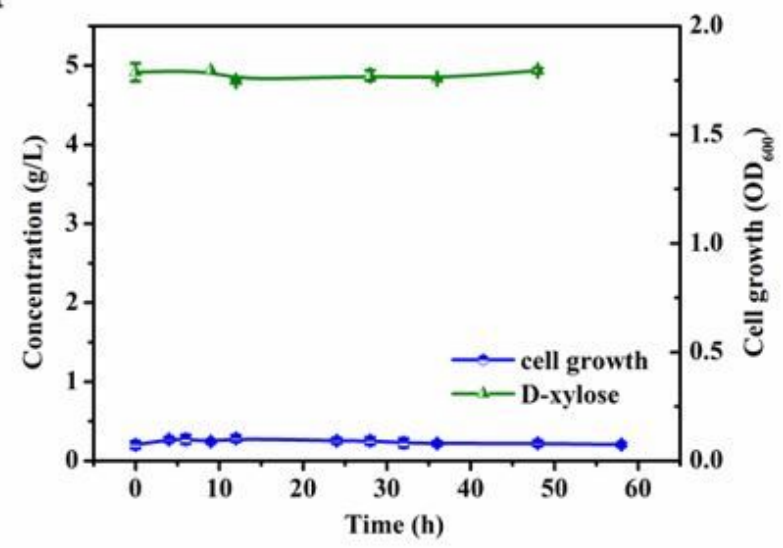

f

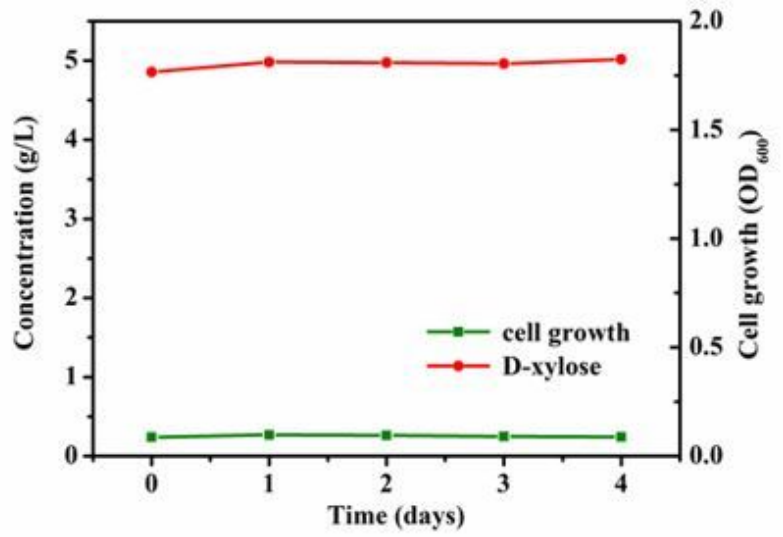


Figure 4

Growth characterization of wild-type P. putida KT2440 and its knockout strains in M9 minimal medium. P. putida KT2440 (a) and P. putida KT2440 pK18MS- $\Delta$ gcd (b) with $5 \mathrm{~g} / \mathrm{L}$ D-glucose; P. putida KT2440 (c) and P. putida KT2440 pK18MS- $\Delta$ gcd (d) with $5 \mathrm{~g} / \mathrm{L}$ D-xylose; P. putida KT2440 pK18MS- $\Delta$ gcd- $\Delta$ gtsABCD with 5 $\mathrm{g} / \mathrm{L}$ D-glucose (e) and $5 \mathrm{~g} / \mathrm{L}$ D-xylose (f). Data shown as mean $\pm \mathrm{SD}$ from at least two independent experiments.

a

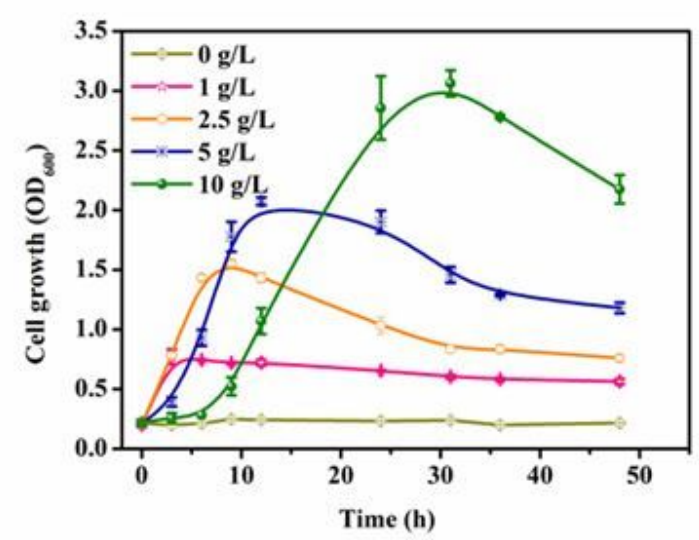

C

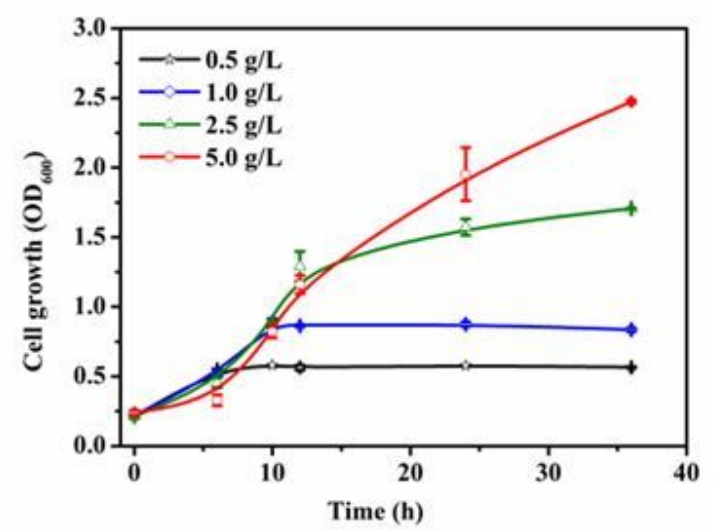

$\mathbf{e}$

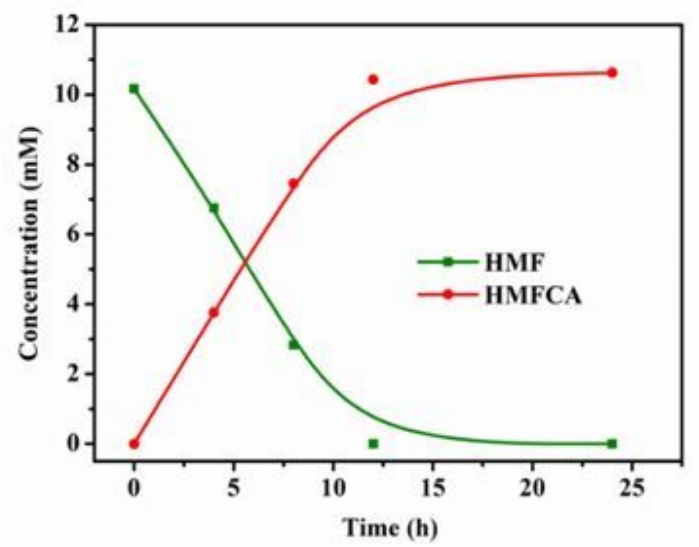

b

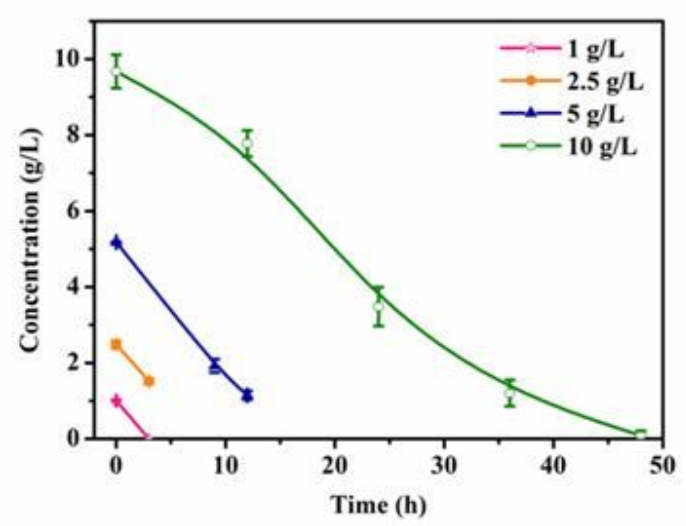

d

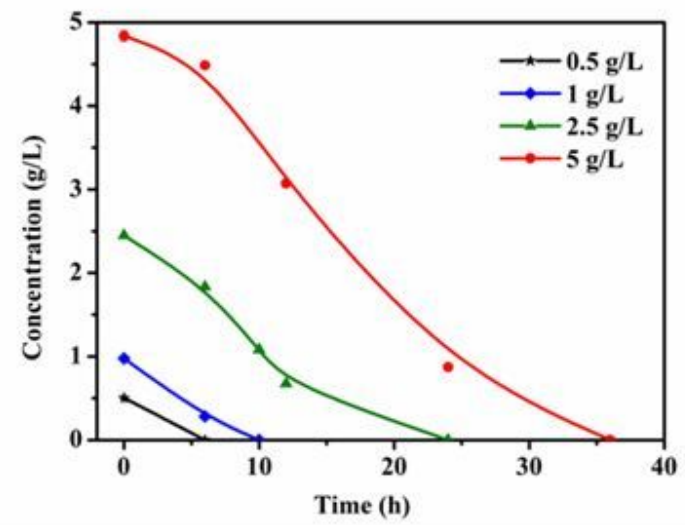

f

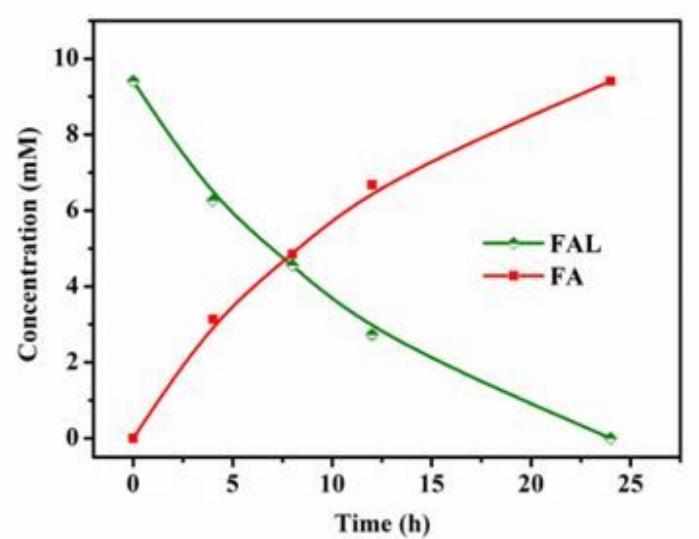

Figure 5 
Inhibitors tolerance analysis of engineered P. putida KT2440 pK18MS- $\Delta$ gcd- $\Delta$ gtsABCD in M9 minimal medium. The cell growth (a) and depletion (b) with different concentrations of sodium acetate; The cell growth (a) and depletion (b) with different concentrations of levulinic acid; The conversion of HMF (e) and FAL (f). Data shown as mean \pm SD from at least two independent experiments.

\section{a}

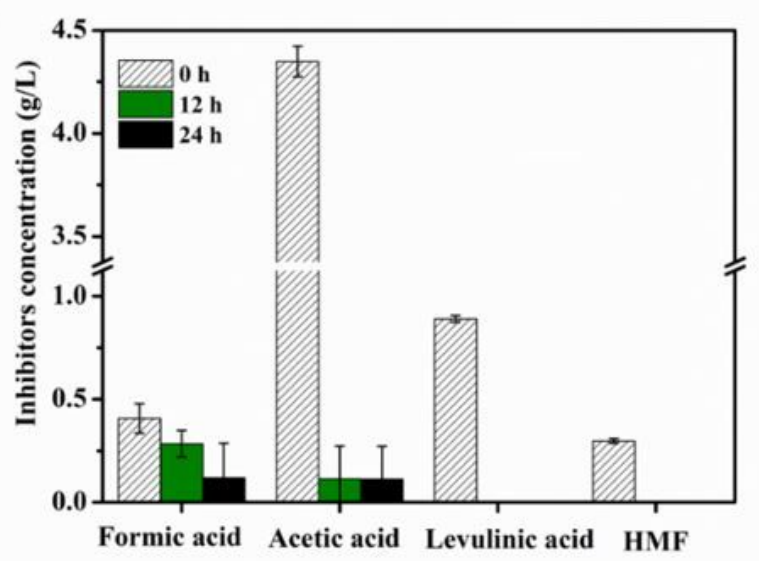

c

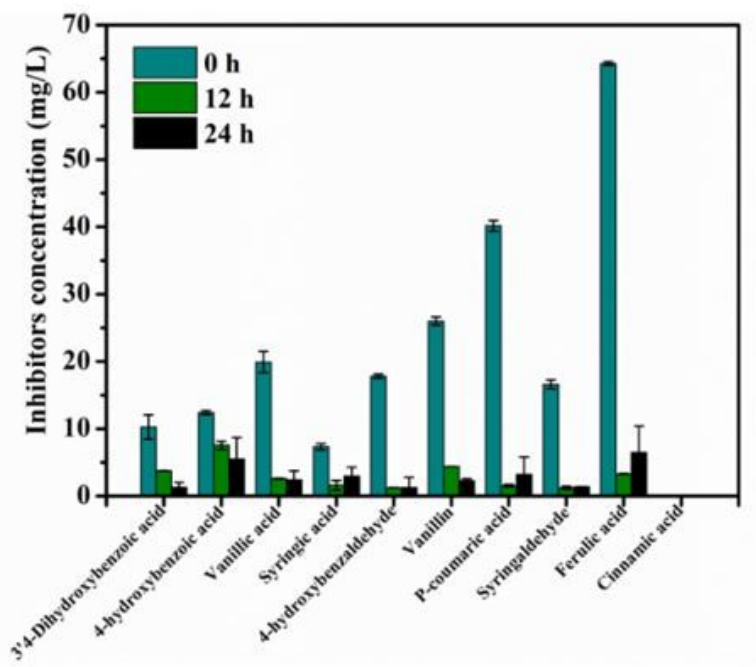

b

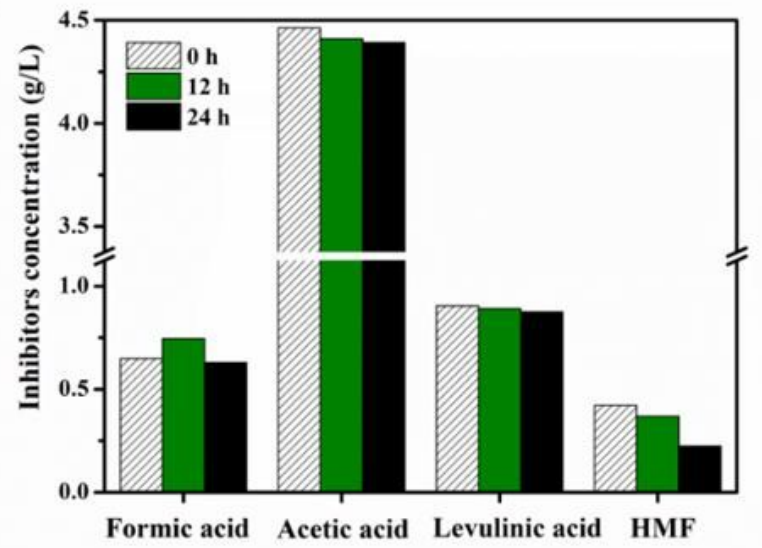

d

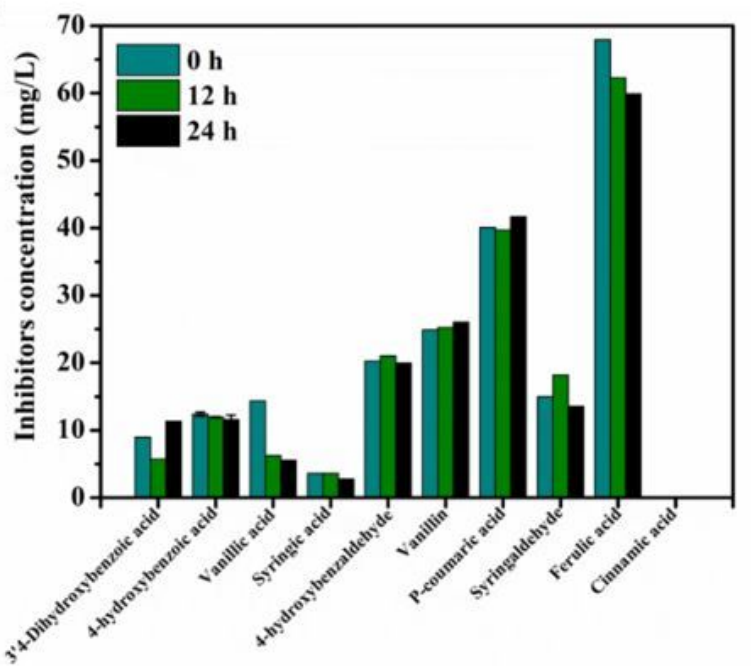

\section{Figure 6}

Comparison of the profiles of inhibitors in undetoxified $30 \%(\mathrm{v} / \mathrm{v})$ hydrolysate by engineered P. putida KT2440 pK18MS- $\Delta$ gcd- $\triangle$ gtsABCD and without detoxified strain (control). The measured parameters include the main inhibitors. The profiles of organic acids and HMF with engineered P. putida (a) and without engineered P. putida (b); The profiles of phenolic compounds with engineered P. putida (c) and without engineered $P$. putida (d). 


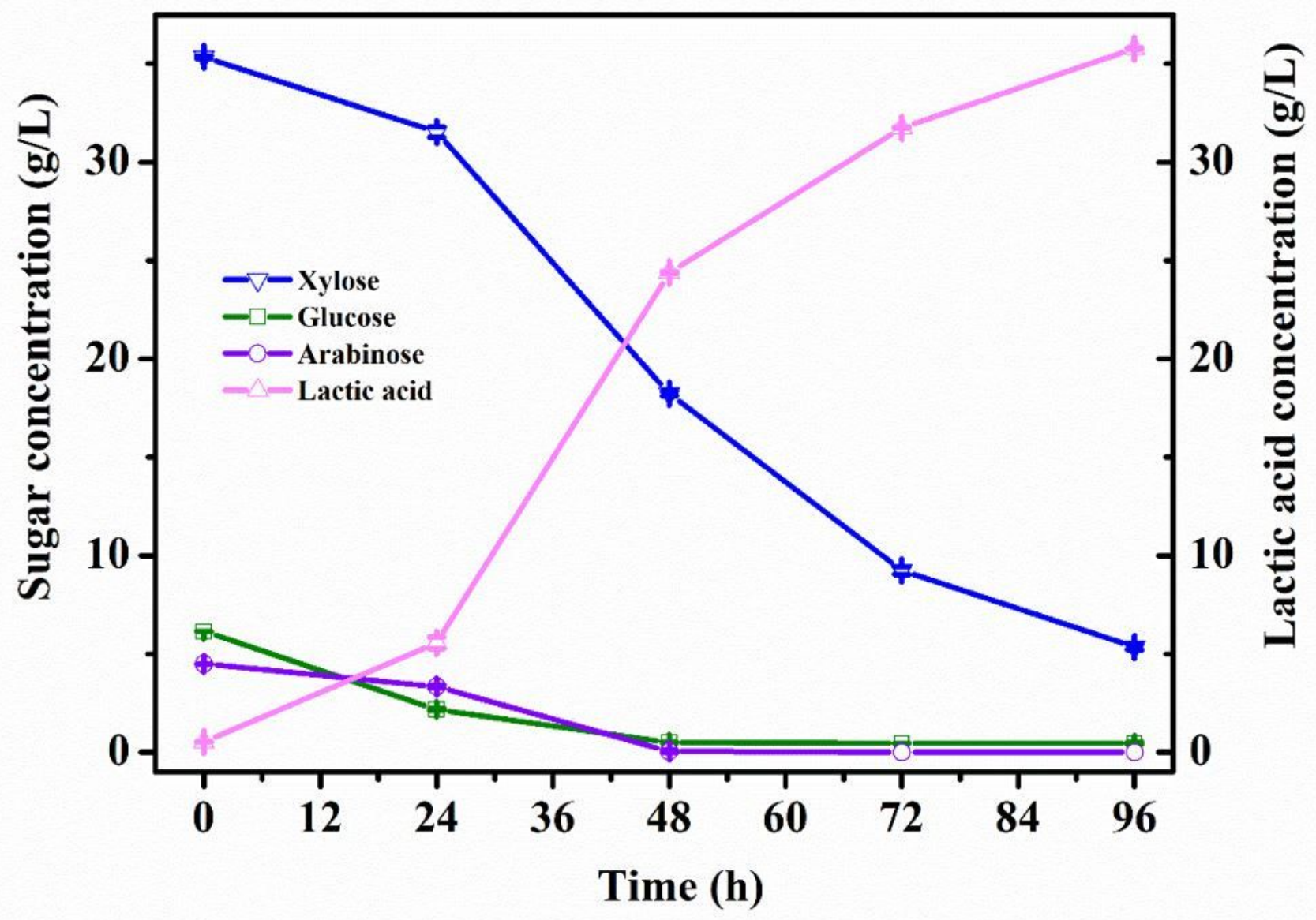

Figure 7

Production of lactic acid from undetoxified $30 \%(\mathrm{v} / \mathrm{v})$ lignocellulosic hydrolysate by using two-bacteria coculture system.

\section{Supplementary Files}

This is a list of supplementary files associated with this preprint. Click to download.

- 20210924FiguretableSI.docx 